Kompass

Neumología

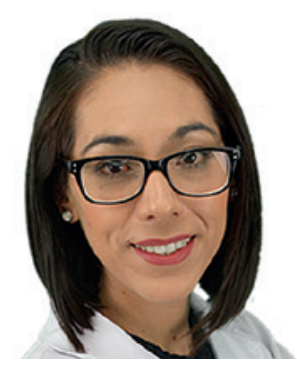

\title{
COVID-19: Una enfermedad multiorgánica
}

\author{
Carla Paola Sánchez-Ríos
}

Neumóloga Clínica, Alta Especialidad Oncología Torácica, Comité Bioseguridad frente a COVID-19, Instituto Nacional de Enfermedades Respiratorias (INER), Ciudad de México, CDMX, México

Resumen de Haberecker M, Schwarz El, Steiger P, et al.: Autopsy-Based Pulmonary and Vascular Pathology: Pulmonary Endotheliitis and Multi-Organ Involvement in COVID-19 Associated Deaths. Respiration 2021 (DOI: 10.1159/000518914).

\section{Keywords}

Adult respiratory distress syndrome · Coronavirus disease-19. Endothelial dysfunction · Multi-organ failure · Respiratory failure

\footnotetext{
Abstract

Background: Findings from autopsies have provided evidence on systemic microvascular damage as one of the underlying mechanisms of Coronavirus disease 2019 (COVID-19). The aim of this study was to correlate autopsy-based cause of death in SARSCoV-2, severe acute respiratory syndrome coronavirus 2 (SARSCoV-2) positive patients with chest imaging and severity grade of pulmonary and systemic morphological vascular pathology.

Methods: Fifteen SARS-CoV-2 positive autopsies with clinically distinct presentations (age 22-89 years) were retrospectively analyzed with focus on vascular, thromboembolic, and ischemic changes in pulmonary and in extrapulmonary sites. Eight patients died due to COVID-19 associated respiratory failure with diffuse alveolar damage in various stages and/or multi-organ failure, whereas other reasons such as cardiac decompensation, complication of malignant tumors, or septic shock were the cause of
}

death in 7 further patients. The severity of gross and histopathological changes was semi-quantitatively scored as 0 (absent), 1 (mild), and 3 (severe). Severity scores between the 2 groups were correlated with selected clinical parameters, initial chest imaging, autopsy-based cause of death, and compared using Pearson $x^{2}$ and Mann-Whitney $U$ tests.

Results: Severe pulmonary endothelitis ( $p=0.031, p=0.029$ ) and multi-organ involvement ( $p=0.026, p=0.006$ ) correlated significantly with COVID-19 associated death. Pulmonary microthrombi showed limited statistical correlation, while tissue necrosis, gross pulmonary embolism, and bacterial superinfection did not differentiate the 2 study groups. Chest imaging at hospital admission did not differ either.

Conclusions: Extensive pulmonary endothelitis and multi-organ involvement are characteristic autopsy features in fatal COVID-19 associated deaths. Thromboembolic and ischemic events and bacterial superinfections occur frequently in SARSCoV-2 infection independently of outcome.

(c) 2021 The Author(s). Published by S. Karger AG, Basel 


\section{Transferencia en la práctica}

\section{Contexto del estudio}

Sabemos que la enfermedad por coronavirus 2019 (coronavirus disease 2019, COVID-19) ha representado un desafío para los sistemas de salud desde diferentes aristas. La insuficiencia respiratoria tipo 1 causada por la infección se ha considerado la principal causa de muerte a pesar de haberse demostrado ya, que esta es una enfermedad multisistémica y que puede activar cascadas inflamatorias con la consecuente afectación y disfunción orgánica a muchos niveles. Se ha documentado que la hipoxia tiene una correlación histopatológica que incluso ha sido analizada con anterioridad en otras afecciones respiratorias y es, el daño alveolar difuso (DAD). Esta manifestación histológica está caracterizada por la formación de membrana hialina intraalveolar, edema y hemorragia seguida de proliferación de fibroblastos. Algo que da particularidad a las manifestaciones de gravedad de la infección SARS-CoV-2 (severe acute respiratory syndrome coronavirus 2 , síndrome respiratorio agudo severo coronavirus 2) al ser comparada con otras infecciones respiratorias es que, la presencia del virus se asocia a cambios microvasculares y trombosis.

Las células endoteliales tienen una participación importante en la patogénesis del síndrome de dificultad respiratoria aguda (SDRA) y de la falla multiorgánica. Se cree que ésto se debe a la inflamación vascular (endotelitis) y a la infiltración de células inflamatorias mediadoras, así como a los cuerpos apoptóticos que se encuentran en diferentes órganos que puede promover un estado procoagulante y así, por diversos mecanismos explicar la hipoxemia desproporcionada respecto a los cambios radiológicos y complicaciones sistémicas. Por último, cabe destacar que además de que, la edad avanzada, la obesidad, la hipertensión y la diabetes mellitus son factores de riesgo de disfunción endotelial y, por lo tanto, podrían explicar el resultado en estos grupos de pacientes y que han sido reportadas alrededor del mundo como comorbilidades asociados a mal pronóstico en pacientes COVID-19.

\section{Resultados del estudio}

Debido a que la interacción entre la necrosis endotelial inducida por virus de SARS-Cov-2, la producción excesiva de citoquinas después del reconocimiento del virus («tormenta de citoquinas») y el síndrome de vasculopatía viral sistémica descrito en varios estudios basados en autopsias y su asociación a daño alveolar difuso como causa de mortalidad más común en enfermos COVID-19, se planteó la necesidad de estudiar la correlación de la causa de muerte basada en autopsias positivas para SARS-CoV-2 con parámetros clínicos y el grado de gravedad de la patología vascular morfológica pulmonar y sistémica.

En este estudio Martina Haberecker y colegas, plantearon varias preguntas, entre ellas: Si la infección por SARS-CoV-2 tiene algún patrón vascular morfológico distinto según la causa de muerte basada en la autopsia o si los eventos tromboembólicos pulmonares y la sobreinfección bacteriana tienen un impacto en la muerte asociada a COVID-19 además de, si algún parámetro clínico podría co- rrelacionarse con los hallazgos morfológicos o la causa de muerte identificada en autopsia de los pacientes. Para dar respuesta a lo anterior, se analizaron los exámenes post mortem de 15 pacientes fallecidos con SARS-CoV-2 positivo en el Instituto de Patología y Patología Molecular del Hospital Universitario de Zúrich, Suiza. La positividad se confirmó mediante rRT-PCR (real-time reverse transcriptase-polymerase chain reaction, reacción en cadena de la polimerasa con transcriptasa inversa en tiempo real) en hisopos nasofaríngeos antes y durante la hospitalización y en 1 paciente mediante análisis post mortem. En todos los pacientes se realizó una autopsia completa que incluyó una evaluación macroscópica y un análisis histológico.

Los diagnósticos y las características histopatológicas en este estudio se llevaron a cabo en un examen macroscópico y utilizando tinciones H\&E (hematoxylin and eosin, hematoxilina y eosina) de rutina en secciones de tejido. Los trombos de fibrina se confirmaron con tinciones de fibrina. En total, se incluyeron 10 pacientes hombres y 5 pacientes mujeres. La edad en el momento de la autopsia osciló entre 22 y 89 años (mediana 72,9 años). Los 15 pacientes fueron hospitalizados (2 a 40 días, mediana de 16,6 días) y sólo 10 pacientes fueron intubados. Las comorbilidades incluyeron en orden descendente de frecuencia: insuficiencia renal crónica, fibrilación auricular, arteriopatía, enfermedad pulmonar intersticial, enfermedad pulmonar obstructiva crónica (EPOC), hipertensión pulmonar, depresión, neoplasias, autoinmunidad y asma.

El examen macroscópico de los pulmones mostró SDRA en 8 casos y mostró un amplio panel de lesiones macroscópicas en 7 casos adicionales. Había grandes zonas hemorrágicas de consolidación y edema bilaterales difusas o focales, parcialmente confluentes, pálidas a amarillentas. Se evidenció infarto pulmonar macroscópico en 6 de 15 casos (40\%), tromboembolismo macroscópico en 5 de 15 casos (30\%). El examen microscópico de los pulmones reveló daño alveolar difuso en diferentes etapas en 8 pacientes (53\%) que fallecieron por COVID-19. Se identificó bronconeumonía en 8 de 15 casos (53\%). Se observó endotelitis de vasos pulmonares de pequeño y mediano calibre en 93\%. Los trombos de fibrina fueron evidentes en $47 \%$ y la oclusión vascular leucocitoclástica en $60 \%$. La extensión de la endotelitis pulmonar se correlacionó significativamente con la causa de muerte por COVID-19 basada en la autopsia (prueba R $p=0,031$, prueba $\cup p=0,029$.

El ARN viral fue evidente en el 30-80\% de los órganos seleccionados en 10 de 15 autopsias, siendo el más alto en muestras de pulmón. El daño vascular en la autopsia no se correlacionó con la extensión de las opacidades pulmonares en vidrio esmerilado. La endotelitis fue un hallazgo frecuente en los pequeños vasos intramiocárdicos y subepicárdicos presentes en todos menos 2 casos (13/15). Los trombos de fibrina fueron menos frecuentes (5 de 15 casos, 30\%) y los trombos leucocíticos en 7 de 15 casos (47\%). A nivel renal, la necrosis tubular aguda se evidenció en el 87\%. En contraste con los pulmones y el corazón, los riñones demostraron endotelitis muy leve. En aquellos pacientes con falla multiorgánica 
(53\%) se encontró necrosis de hepatocitos centrolobulillares en parches y necrosis confluente asociada con trombos de fibrina en los respectivos vasos. La necrosis del intestino delgado se documentó en el $27 \%$, lo que condujo al escenario de falla multiorgánica.

La endotelitis pulmonar grave (R-test $p=0,031, \mathrm{U}$ test $p=0,029)$ y la afectación multiorgánica de COVID-19 (R-test $p=0,026, \mathrm{U}$ test $p$ $=0,006$ ) se correlacionaron significativamente con la muerte evidenciando que la endotelitis pulmonar de grado grave, la afectación multiorgánica de la endotelitis y la necrosis tisular se correlacionan significativamente con deceso en enfermos COVID-19.

A pesar de lo que documenta la literatura, en esta cohorte, ninguno de los puntajes SOFA (sequential organ failure assessment, evaluación secuencial de la insuficiencia de órganos) se correlacionó con la causa de muerte en COVID-19 pudiendo influir que la extensión del daño endotelial descrito en este estudio es más prominente en el pulmón, mientras que las manifestaciones extrapulmonares son menos evidentes.

\section{Conclusiones y recomendaciones para la práctica}

Si bien, al día de hoy se cuenta con varias publicaciones de pacientes COVID-19 estudiados post mortem, este estudio muestra una clara relación entre daño endotelial pulmonar con daño orgánico y muerte en pacientes infectados. Considero que es sumamente importante dejar de ver a la enfermedad COVID-19 en un contexto minoritario de gravedad o bien, sólo como una neumonía pues hasta hace algunos meses seguía siendo una práctica común tanto por el personal médico como no médico, lo que probablemente impactó de manera negativa a la toma de medidas de salud pública con retardo, para el control de la pandemia.
Tomando en cuenta lo anteriormente mencionado sugiero:

1 Asociar al virus de SARS-CoV-2 no sólo con daño pulmonar sino con un potencial de afectación multiorgánica.

2 Pacientes con antecedentes de comorbilidades asociadas a daño endotelial deben ser evaluados de manera cautelosa y si es posible mediante las aplicaciones de score clínicos que evalúen por sus estados comórbidos, el riesgo de deterioro a corto plazo.

3 Tener en mente que el daño pulmonar evidenciado por extensión tomográfica y gasométricamente por gravedad de SDRA, tiene relación con la falla multiorgánica y pobre pronóstico en enfermos COVID-19.

4 Considerar que la hipoxia en estos pacientes es multifactorial por lo que pacientes con requerimiento de oxígeno deben ser evaluados para descartar complicaciones vasculares pues el daño alveolar, se asocia a microtrombosis y potencial afectación de circulación pulmonar que puede empeorar la IR tipo 1 ameritando otras terapias a parte de los esteroides y aumento de fracciones inspiradas de oxígeno.

5 Siempre que sea posible, pacientes con hipoxemia debiesen atenderse y evaluarse multidisciplinariamente en contexto hospitalario.

\section{Disclosure Statement}

No existen conflictos de interés con respecto a esta transferencia de conocimientos.

Correspondencia:

Dra. Carla Paola Sánchez-Ríos, pao1144tost@gmail.com 